\title{
ORIENTAÇÃO INDIRETA DE IMAGENS DIGITAIS USANDO TELHADOS DE DUAS ÁGUAS COMO APOIO DE CAMPO
}

\author{
Indirect image orientation using gable roofs as ground control \\ NADISSON LUIS PAVAN ${ }^{1}$ \\ DANIEL RODRIGUES DOS SANTOS ${ }^{1}$ \\ ALUIR PORFÍRIO DAL POZ ${ }^{2}$ \\ ${ }^{1}$ Universidade Federal do Paraná \\ Setor de Ciências da Terra \\ Programa de Pós-Graduação em Ciências Geodésicas \\ CEP 81531-Curitiba/PR-Brasil \\ nadissonlp@hotmail.com;danielsantos@ufpr.br \\ ${ }^{2}$ Universidade Estadual Paulista \\ Faculdade de Ciências e Tecnologia \\ Programa de Pós-Graduação em Ciências Cartográficas \\ CEP 19060-900-Presidente Prudente/SP-Brasil \\ aluir@fct.unesp.br
}

\begin{abstract}
RESUMO
Este trabalho apresenta um método para orientação indireta de imagens digitais usando telhado de duas águas como apoio de campo. Edificações com telhados de duas águas são visualmente selecionados pelo operador na imagem digital e suas correspondentes na imagem de intensidade do pulso laser. Um ponto qualquer sobre as cumeeiras de cada uma das edificações é manualmente selecionado na imagem digital. Os telhados adjacentes das edificações correspondentes são manualmente delimitados, os erros espúrios são automaticamente filtrados e em seguida, os planos dos telhados são segmentados e ajustados pelo MMQ. Finalmente, os pontos imagem coletados sobre a cumeeira das edificações e os parâmetros dos planos dos telhados adjacentes são usados para estimativa dos parâmetros de orientação exterior da câmera, aplicando o modelo matemático proposto. Este trabalho descreve em detalhes o modelo matemático proposto e discute as vantagens e desvantagens da abordagem apresentada comparada com o método convencional de orientação de imagens. Experimentos foram realizados e os resultados obtidos
\end{abstract}


mostraram a potencialidade do método.

Palavras-chave: Integração de Dados; Sistema LiDAR Aerotransportado; Orientação Indireta de Imagens; Telhados de Duas Águas; Mmodelo Fotogramétrico.

\section{ABSTRACT}

This article presents an indirect method for image orientation using gable roofs derived from Airborne Light Detection and Ranging. Appropriate gable roofs in the intensity pulse image, is visualy selected by the operator. Thus, the regions of corresponding neighbouring planes are visually delimited and adjusted by the use of least-squares adjustment method being computed the parameters of the planes. In the digital image, the corresponding gable roofs are visually selected and for each ridge line defined between the neighbouring planes, any one intermediate point is collected. Finally, both parameters of the planes and intermediate points are used for determination of exterior orientation parameters of camera by using the proposed model with the conventional least-squares adjustment procedure. The paper describes the derivation of proposed mathematical model in detail and discusses the advantages and disadvantages of the approach compared to the conventional surveying one. The results obtained showed that the proposed model is likely to work out.

Keywords: Data Integration; Airborne Light Detection and Ranging; Gabled Roofs; Indirect Image Orientation.

\section{INTRODUÇÃO}

A orientação de imagens é tarefa fundamental em Fotogrametria e consiste em determinar os parâmetros de orientação exterior (posição e rotação) da câmera no momento da exposição das imagens. O método direto consiste em determinar a posição e rotação da câmara a partir da integração de sensores GPS (em inglês, global positioning system) e INS (em inglês, inertial navegation system). No método indireto um modelo fotogramétrico, como o de colinearidade, e um método de ajustamento de observações, tal como o Método dos Mínimos Quadrados (MMQ) são aplicados para estimar os parâmetros de orientação exterior da câmera (POEs). Um terceiro método, conhecido como híbrido, pode ser empregado para refinar os POEs obtidos com a tecnologia de integração de sensores GPS/INS (método direto), através de apoio de campo e uso de um modelo fotogramétrico rigoroso (método indireto).

Desde que uma nuvem de pontos tridimensionais derivada do sistema LiDAR aerotransportado (em inglês, Airborne Light Detection and Ranging) está disponível, não se pode garantir a correspondência ponto a ponto entre o conjunto de dados fotogramétricos e o sistema LiDAR aerotransportado (SLA). Uma vez que a densidade de pontos perfilados sobre a superfície física é dada em função da velocidade de cruzeiro da aeronave, comprimento da faixa e altura de vôo, 
mecanismo e ângulo de varredura, bem como da frequência de repetição do pulso, não é possível controlar todos os fatores supracitados. Desta forma, o perfilamento é executado de forma irregular, implicando em uma amostragem semi-aleatória do terreno. Além disso, as feições pontuais exigem pré-sinalização de alvos, não são facilmente identificadas e semanticamente reconhecidas em imagens de intensidade do pulso laser, e também são ocorrências isoladas, cujas desvantagens recaem sobre o processo de estabelecimento automático de correspondências.

Por outro lado, as feições lineares e os planos segmentados são primitivas potenciais que podem ser consideradas como uma alternativa mais adequada no processo de orientação de imagens, quando comparadas com feições pontuais e considerando a integração de dados fotogramétricos e LIDAR. Na imagem digital, as feições lineares são facilmente extraídas e identificadas. Enquanto na imagem de intensidade do pulso LASER, feições lineares podem ser determinadas por meio da intersecção de planos adjacentes, o que implica em uma linha reta geometricamente bem definida. Neste caso, o problema de orientação não exige correspondência ponto a ponto e pré-sinalização de alvos.

Lugnani (1980) demonstrou a viabilidade da utilização de entidades lineares como controle de campo no processo de orientação indireta de imagens. Mulawa e Mikhail (1988) incluíram diferentes modelos matemáticos (baseada em linhas, curvas e planos) e apresentou o princípio de isometria com feições lineares. Tommaselli e Lugnani (1988) apresentaram o modelo dos planos equivalentes, que estabelece uma relação funcional entre linhas retas no espaço objeto e no espaço imagem. Tommaselli e Tozzi (1996) aperfeiçoaram o modelo supracitado para aplicá-lo num procedimento recursivo de resseção espacial de imagens.

Aproveitando o impacto do conceito de integração de dados advindos de diferentes sensores Habib et. al. (2004) apresentaram uma abordagem para orientação indireta de imagens utilizando feições free forms e dados SLA. Dalmolin et. al. (2005) utilizaram o modelo dos planos equivalentes, apresentado por Tommaselli e Tozzi (1996), cujo apoio de campo é obtido através de linhas retas extraídas automaticamente na imagem de intensidade do pulso laser. Para mostrar a viabilidade do uso de planos segmentados no método indireto de orientação de imagens Habib et al. (2005) e Habib et al. (2007) apresentaram uma nova abordagem onde superfícies planas, especificamente telhados de edificações definidas através de dados brutos derivados do SLA são utilizadas como apoio de campo no georreferenciamento de imagens digitais.

Dentro deste novo paradigma de integração de dados derivados de diferentes fontes de aquisição de informações espaciais, este trabalho tem como proposta apresentar um modelo fotogramétrico fundamentado em telhados de duas águas como apoio de campo para a orientação indireta de imagens digitais. O modelo proposto é baseado no relacionamento funcional entre planos de telhados adjacentes extraídos de edificações derivadas do SLA (espaço objeto) e qualquer ponto imagem da cumeeira de uma edificação presente na imagem digital (espaço 
imagem). Após a segmentação dos planos seus parâmetros são determinados e utilizados como apoio de campo no processo de orientação de imagens.

Este trabalho está organizado da forma como segue: o método proposto é apresentado na próxima seção, seguido pelos experimentos e discussões dos resultados; a última seção apresenta as principais conclusões e recomendações para trabalhos futuros.

\section{MÉTODO}

O método proposto está dividido em três etapas básicas, a saber: (1) Edificações com telhados de duas águas são visualmente selecionados pelo operador na imagem digital e suas correspondentes na imagem de intensidade do pulso laser. Um ponto qualquer sobre as cumeeiras de cada uma das edificações é manualmente selecionado na imagem digital; (2) Os telhados de duas águas das edificações correspondentes, na imagem de intensidade do pulso laser, são manualmente delimitados. Em seguida, os erros espúrios são automaticamente filtrados e os planos dos telhados são segmentados e ajustados pelo MMQ; (3) Finalmente, os pontos imagem coletados sobre a cumeeira das edificações e os parâmetros dos planos dos telhados adjacentes são usados para estimativa dos parâmetros de orientação exterior da câmera, aplicando o modelo matemático que será proposto.

\subsection{Extração e segmentação dos planos adjacentes}

As edificações usadas como apoio de campo são previamente selecionadas na imagem de intensidade do pulso laser. Os telhados de edificações que mais se enquadram na metodologia proposta são do tipo duas águas. Quatro cantos ao redor de uma “água” de cada uma das edificações são manualmente coletados, sendo posteriormente aplicado um algoritmo de filtragem de dados espúrios e segmentação automática de planos (Figura 1). O algoritmo de filtragem é aplicado para eliminar pontos tridimensionais que não pertencem ao plano que define o telhado da edificação selecionada, tais como: pontos sobre lareiras, antenas de televisão etc.

É importante notar que os pixels coletados nos cantos das edificações selecionadas pelo operador estão referenciados ao sistema digital (Coluna, Linha). Sendo assim, é necessário transformar suas coordenadas para o sistema referencial geodésico que se encontra a nuvem de pontos derivada do sistema LiDAR. A partir desta transformação é necessário encontrar, no arquivo bruto dos dados SLA, a coordenada altimétrica correspondente. Para maiores detalhes ver Dalmolin et al. (2005).

A figura 1 mostra um exemplo de edificação com telhados de duas águas na imagem de intensidade do pulso laser, os pixels coletados na imagem de intensidade do pulso, duas edificações segmentadas e um ponto qualquer da cumeeira de uma edificação selecionado na imagem digital. 
Figura 1 - (A) Edificação selecionada na imagem de intensidade laser. (B) Exemplos de edificações segmentadas. (C) Ponto qualquer da cumeeira de um telhado no espaço imagem.

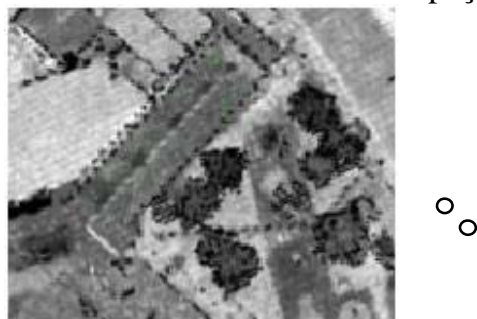

(A)
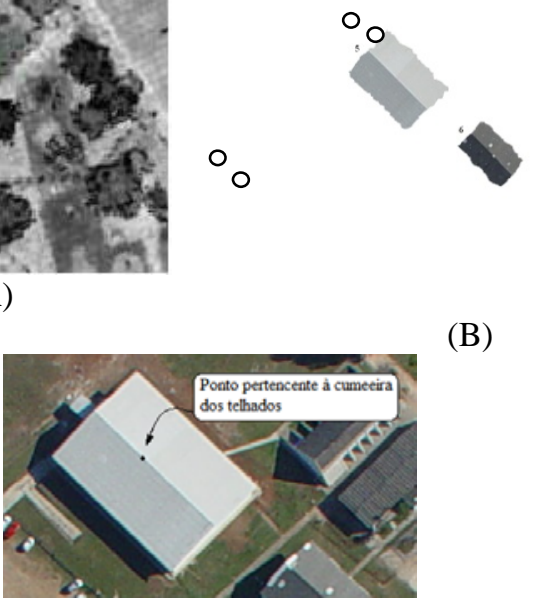

(B)

(C)

Os parâmetros dos planos dos telhados adjacentes das edificações segmentadas são calculados usando o MMQ. No espaço imagem, a cumeeira de uma edificação pode ser representada por uma seqüência de pontos bidimensionais (ver Figura 1C). No método proposto, qualquer ponto imagem que representa a cumeeira selecionada na imagem digital é manualmente coletado e transformado para o sistema referencial fotogramétrico, cujas coordenadas são devidamente corrigidas dos efeitos sistemáticos.

\subsection{Estratégia de intersecção de telhados adjacentes}

Geralmente, uma reta no espaço euclidiano é determinada por dois pontos, por um ponto e seu vetor diretor ou pela intersecção entre dois planos. No último caso, se pode obter uma equação matemática através da intersecção de duas faces planas, cujos atributos são representados, neste caso, por telhados adjacentes. Uma edificação do tipo telhado de duas águas é definida por dois telhados adjacentes, cujos planos $\pi_{1}$ e $\pi_{2}$ são definidos pela equação geral do plano,

$$
\begin{aligned}
& A_{1} X+B_{1} Y+C_{1} Z+D_{1}=0 \\
& A_{2} X+B_{2} Y+C_{2} Z+D_{2}=0
\end{aligned}
$$

sendo, $A_{1}, B_{1}, C_{1}$ os parâmetros do plano $\pi_{1}$ e $A_{2}, B_{2}, C_{2}$ os parâmetros do plano $\pi_{2}$. 
Logo os vetores ortogonais aos planos $\left(\overrightarrow{v_{1}}\right.$ e $\left.\overrightarrow{v_{2}}\right)$ podem ser expressos em relação aos sistemas de coordenadas, como segue:

$$
\begin{aligned}
& \overrightarrow{v_{1}}=\left[\begin{array}{lll}
A_{1} & B_{1} & C_{1}
\end{array}\right]^{T} \\
& \overrightarrow{v_{2}}
\end{aligned}=\left[\begin{array}{lll}
A_{2} & B_{2} & C_{2}
\end{array}\right]^{T}
$$

Sendo, $\vec{d}$ o vetor diretor da reta $\overleftrightarrow{P Q}$, então $\vec{d}$ é simultaneamente ortogonal aos vetores $\overrightarrow{v_{1}}$ e $\overrightarrow{v_{2}}$ normais aos planos (Figura 2). Neste caso, $\vec{d}$ pode ser determinado pelo produto vetorial entre $\overrightarrow{v_{1}}$ e $\overrightarrow{v_{2}}$ como segue:

$$
\vec{d}=\overrightarrow{v_{1}} \times \overrightarrow{v_{2}}=\left[\begin{array}{l}
A_{1} \\
B_{1} \\
C_{1}
\end{array}\right] \times\left[\begin{array}{l}
A_{2} \\
B_{2} \\
C_{2}
\end{array}\right]=\left[\begin{array}{l}
B_{1} C_{2}-C_{1} B_{2} \\
C_{1} A_{2}-A_{1} C_{2} \\
A_{1} B_{2}-B_{1} A_{1}
\end{array}\right]=\left[\begin{array}{c}
l \\
m \\
n
\end{array}\right]
$$

Figura 2 - Edificação com telhados de duas águas e elementos geométricos no espaço objeto.

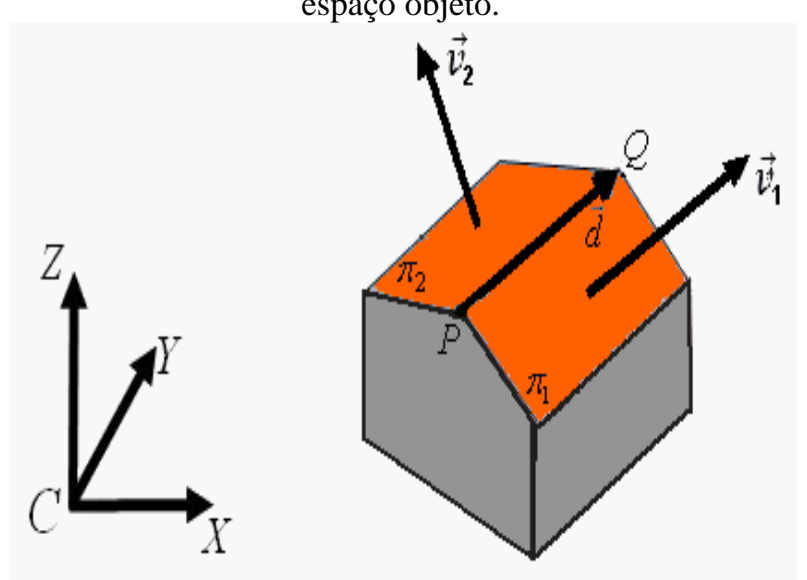

Como o ponto $P$ determinado pelas coordenadas $\left(X_{P}, Y_{P}, Z_{P}\right)$ pertence aos planos $\pi_{1}$ e $\pi_{2}$, tem-se o seguinte sistema de equações lineares a saber:

$$
\left\{\begin{array}{l}
A_{1} X_{P}+B_{1} Y_{P}+C_{1} Z_{P}=-D_{1} \\
A_{2} X_{P}+B_{2} Y_{P}+C_{2} Z_{P}=-D_{2}
\end{array}\right.
$$

Este sistema de equações lineares pode ser reescrito pelo produto escalar dos vetores, como segue: 


$$
\left\{\begin{array}{l}
\overrightarrow{v_{1}} \cdot \vec{u}=\left[\begin{array}{l}
A_{1} \\
B_{1} \\
C_{1}
\end{array}\right]\left[\begin{array}{lll}
X_{P} & Y_{P} & Z_{P}
\end{array}\right]^{T}=-D_{1} \\
\overrightarrow{v_{2}} \cdot \vec{u}=\left[\begin{array}{l}
A_{2} \\
B_{2} \\
C_{2}
\end{array}\right]\left[\begin{array}{lll}
X_{P} & Y_{P} & Z_{P}
\end{array}\right]^{T}=-D_{2}
\end{array}\right.
$$

sendo, $\vec{u}=\left[\begin{array}{lll}X_{P} & Y_{P} & Z_{P}\end{array}\right]^{T}$ o vetor que parte da origem do sistema coordenadas tridimensionais no espaço objeto ao ponto $P$ qualquer e $D_{1}$ e $D_{2}$ são os termos independentes utilizados no ajustamento dos planos.

\subsection{Modelo proposto}

O modelo proposto é baseado no relacionamento funcional entre planos adjacentes que representam os telhados de edificações derivadas do SLA (espaço objeto) e qualquer ponto imagem da cumeeira de uma edificação presente na imagem digital (espaço imagem). Especificamente, o vetor diretor da reta no espaço objeto e o ponto $P$, (Figura 3), são substituídos, no modelo matemático, pelos parâmetros dos planos representados pela intersecção dos telhados adjacentes. A Fig. 3 mostra a geometria do modelo proposto.

Figura 3 - Geometria do modelo proposto.

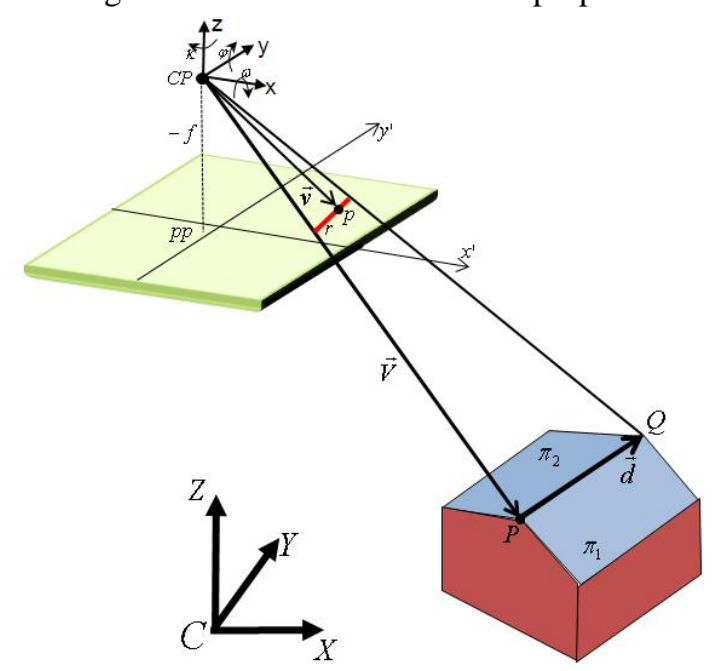

Os seguintes elementos ilustrados na Figura 1 são definidos, como segue: CP é o centro perspectivo da câmara; CPxyz é o sistema referencial fotogramétrico tridimensional cuja origem é o centro perspectivo da câmara; $f$ é a distância focal calibrada da câmara; $o x^{\prime} y^{\prime}$ é o sistema de coordenadas com origem no centro da 
imagem; $\kappa, \varphi$ e $\omega$ são os ângulos de Euler correspondentes às rotações em torno de $z, y$ e $x$, respectivamente; $p p$ é o ponto principal definido pela projeção ortogonal do CP sobre o plano da imagem; $r$ é a linha reta no espaço imagem homóloga a reta $\overleftrightarrow{P Q} ; p$ é um ponto qualquer na linha reta $r$ no espaço imagem; $\vec{V}$ é o vetor de coordenadas do CP no referencial do espaço objeto; e $\vec{v}$ é o vetor de coordenadas do ponto no sistema referencial fotogramétrico.

$\mathrm{O}$ vetor $\vec{v}$ multiplicado pela matriz de rotação transposta $R^{T}$ (dada em função dos ângulos de Euler $(\kappa, \varphi, \omega)$ (sendo essa matriz o produto das matrizes na ordem é $R_{\kappa}, R_{\varphi}$ e $R_{\omega}$ ) é coplanar aos vetores $\vec{d}$ e $\vec{V}$. Assim, o produto misto desses vetores deve ser zero, como segue:

$$
(\vec{V} \times \vec{d}) \cdot \vec{v}=0
$$

Os vetores $\vec{V}$ e $\vec{v}$ são expressos em relação ao sistema referencial fotogramétrico e ao sistema referencial do espaço objeto, a saber:

$$
\begin{gathered}
\vec{V}=\left[\begin{array}{c}
\Delta X \\
\Delta Y \\
\Delta Z
\end{array}\right]=\left[\begin{array}{c}
X_{P}-X_{0} \\
Y_{P}-Y_{0} \\
Z_{P}-Z_{0}
\end{array}\right] \\
\vec{v}=\left[\begin{array}{c}
\Delta x \\
\Delta y \\
-f
\end{array}\right]=R^{T}\left[\begin{array}{c}
x_{i}^{\prime}-x_{0} \\
y_{i}^{\prime}-y_{0} \\
-f
\end{array}\right]
\end{gathered}
$$

sendo, $X_{0}, Y_{0}, Z_{0}$ são coordenadas do CP da câmera, $x_{i}^{\prime}, y_{i}^{\prime}$ coordenadas de um ponto qualquer da cumeeira no espaço imagem e $x_{0}, y_{0}$ são as coordenadas do ponto principal.

Deste modo o vetor $\vec{V}$ pode ser escrito pela soma dos vetores $\overrightarrow{V_{P}}$ e $\overrightarrow{V_{0}}$, assim sendo:

$$
\vec{V}=\vec{u}+\overrightarrow{V_{0}}=\left[\begin{array}{l}
X_{P} \\
Y_{P} \\
Z_{P}
\end{array}\right]+\left[\begin{array}{l}
-X_{0} \\
-Y_{0} \\
-Z_{0}
\end{array}\right]
$$

Substituindo a Equação (11) na Equação (8), tem-se:

$$
\left(\left(\vec{u}+\overrightarrow{V_{0}}\right) \times \vec{d}\right) \cdot \vec{v}=0
$$

Utilizando as propriedades de produto escalar (ou produto interno) descrita tem-se: 


$$
\left(\vec{u} \times \vec{d}+\overrightarrow{V_{0}} \times \vec{d}\right) \cdot \vec{v}=0
$$

Substituído a Equação (5) na Equação (13) tem-se:

$$
\left(\vec{u} \times\left(\overrightarrow{v_{1}} \times \overrightarrow{v_{2}}\right)+\left(\overrightarrow{V_{0}} \times \vec{d}\right)\right) \cdot \vec{v}=0
$$

A Relação entre produto vetorial de três vetores é dada pela relação:

$$
\vec{u} \times\left(\overrightarrow{v_{1}} \times \overrightarrow{v_{2}}\right)=\left(\vec{u} \cdot \overrightarrow{v_{2}}\right) \overrightarrow{v_{1}}-\left(\vec{u} \cdot \overrightarrow{v_{1}}\right) \overrightarrow{v_{2}}
$$

Assim substituído a Equação (15) na Equação (14) tem-se:

$$
\left(\left(\vec{u} \cdot \overrightarrow{v_{2}}\right) \overrightarrow{v_{1}}-\left(\vec{u} \cdot \overrightarrow{v_{1}}\right) \overrightarrow{v_{2}}+\left(\overrightarrow{V_{0}} \times \vec{d}\right)\right) \cdot \vec{v}=0
$$

Substituído a Equação (7) na Equação (16) tem-se:

$$
\left(-D_{2} \overrightarrow{v_{1}}+D_{1} \overrightarrow{v_{2}}+\left(\overrightarrow{V_{0}} \times \vec{d}\right)\right) \cdot \vec{v}=0
$$

Resolvendo o produto misto, tem-se o modelo dos planos adjacentes proposto neste trabalho, a saber:

$$
\begin{aligned}
& {\left[\left(D_{1} A_{2}-A_{1} D_{2}\right)-Y_{0}\left(A_{1} B_{2}-A_{2} B_{1}\right)+Z_{0}\left(A_{2} C_{1}-A_{1} C_{2}\right)\right]\left(r_{11} \Delta x+r_{21} \Delta y-r_{31} f\right) } \\
+ & {\left[\left(D_{1} B_{2}-B_{1} D_{2}\right)-Z_{0}\left(B_{1} C_{2}-B_{2} C_{1}\right)+X_{0}\left(A_{1} B_{2}-A_{2} B_{1}\right)\right]\left(r_{12} \Delta x+r_{22} \Delta y-r_{32} f\right) \quad(18) } \\
+ & {\left[\left(D_{1} C_{2}-C_{1} D_{2}\right)-X_{0}\left(A_{2} C_{1}-A_{1} C_{2}\right)+Y_{0}\left(B_{1} C_{2}-B_{2} C_{1}\right)\right]\left(r_{13} \Delta x+r_{23} \Delta y-r_{33} f\right)=0 }
\end{aligned}
$$

sendo, $r_{11}, \ldots r_{33}$ são os elementos da matriz de rotação.

A equação (18) pode ser usada para estimar os parâmetros de orientação exterior da câmera. Entretanto, a equação mencionada não é linear em relação aos parâmetros e observações. Por isso, se deve linearizar o modelo proposto usando série de Taylor e uma abordagem iterativa é necessária para solucionar o problema. O modelo dos planos adjacentes é composto por apenas uma equação de condição, cujos parâmetros estão implícitos na equação sendo necessário o uso do modelo combinado de ajustamento de observações. É importante verificar que para cada ponto coletado na imagem digital ter-se-á uma equação de condição (equação 18), composta por 10 observações $\left(x_{i}^{\prime}-x_{0}, y_{i}^{\prime}-y_{0}, A_{1}, B_{1}, C_{1}, D_{1}, A_{2}, B_{2}, C_{2}, D_{2}\right)$ e por 6 parâmetros $\left(\kappa, \varphi, \omega, X_{0}, Y_{0}, Z_{0}\right)$ a serem estimados pelo MMQ.

\section{EXPERIMENTOS E ANÁLISE DOS RESULTADOS}

O seguinte conjunto de dados foi utilizado: uma imagem digital com GSD de 0,4m, tomada com uma câmera digital de pequeno formato Sony DSC F717, cujos 
parâmetros de orientação interior (POIs) foram previamente determinados por um processo de auto-calibração de câmeras (CLARKE e FRAYER, 1998; BROWN, 1971); um conjunto de 19 pontos tridimensionais, devidamente pré-sinalizados e determinados por levantamento GPS $\left(\sigma_{\mathrm{XY}}=0,05 \mathrm{~m}\right)$; nuvem de pontos tridimensionais derivados do SLA, com perfilamento executado pelo sistema da OPTECH ALTM 2050, altura média de $975 \mathrm{~m}$ e densidade de 2,24 pontos $/ \mathrm{m}^{2}(\sim 0,7$ $\mathrm{m}$ espaçamento entre os pontos). De acordo com as especificações de voo a precisão planialtimétrica dos dados SLA esperada é de $0,80 \mathrm{~m}$ e $0,25 \mathrm{~m}$, respectivamente; e uma imagem de intensidade do pulso laser derivada do SLA. Os algoritmos utilizados nos experimentos foram implementados em linguagem $\mathrm{C}++$ ambiente de programação Builder 5.0 para Windows.

Para avaliar a potencialidade do método proposto foi aplicada a resseção espacial de uma imagem adquirida com câmera digital de pequeno formato. Os testes realizados foram divididos em quatro experimentos, como segue: a) oito edificações extraídas da imagem de intensidade do pulso laser; b) vinte e um edificações bem distribuídas e de pequena dimensão; c) vinte e cinco edificações bem distribuídas e de grande dimensão; e d) quarenta e seis edificações bem distribuídas. A Tabela I mostra o conjunto de experimentos elaborados e a Fig. 4 apresenta a distribuição e tamanho das edificações segmentadas na imagem de itensidade do pulso laser.

Tabela 1 - Conjunto de experimentos elaborados com dados reais.

\begin{tabular}{c|c}
\hline \# edificações & \# Experimentos \\
\hline 8 & I (Fig. 8a) \\
\hline 21 & II (Fig. 8b) \\
\hline 25 & III (Fig. 8c) \\
\hline 46 & IV (Fig. 8d) \\
\hline
\end{tabular}

Para a estimação dos POEs usando o MMQ, juntamente com o modelo matemático proposto neste trabalho, os experimentos realizados devem seguir as seguintes etapas, a saber: cada edificação é manualmente selecionada e segmentada na imagem de intensidade do pulso laser; os parâmetros dos planos de cada edificação são calculados usando o MMQ, juntamente com a equação geral do plano; na imagem digital, são manualmente coletados qualquer ponto imagem presente na cumeeira de cada edificação correspondente no espaço imagem; e o método proposto é aplicado para estimação dos POEs. 
Figura 4 - Edificações selecionadas e segmentadas. (A) Experimento I; (B) Experimento II; (C) Experimento III; (D) Experimento IV.
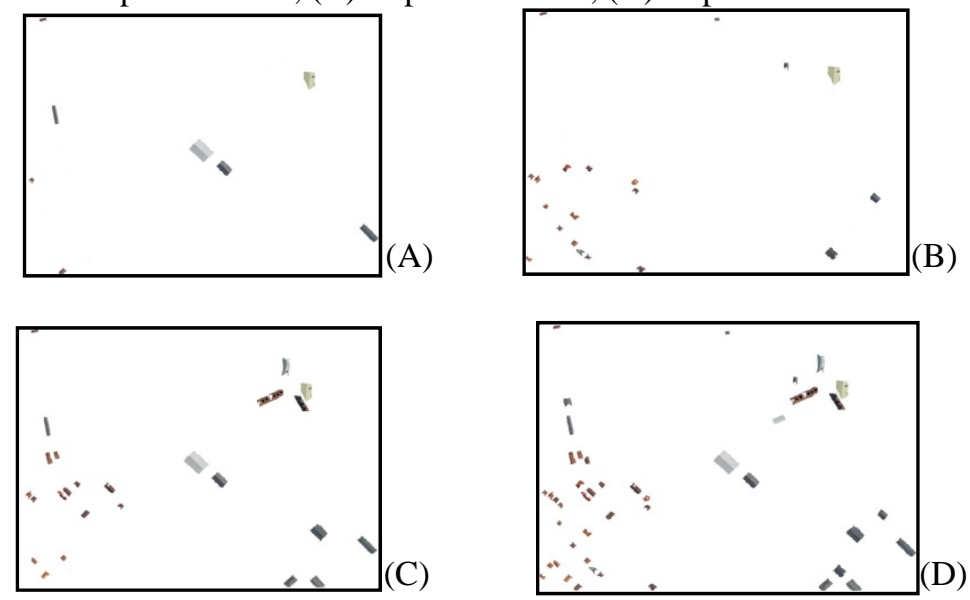

Para avaliar os resultados obtidos com o método proposto, o método convencional de resseção espacial de imagens foi aplicado incorporando um conjunto de 11 pontos tridimensionais, devidamente pré-sinalizados e determinados através de levantamento GPS (precisão XYZ de 0,05 m). Os parâmetros de orientação exterior determinados com o método convencional serão considerados como valores de referência. Na Tabela II podem ser encontrados os valores dos parâmetros estimados por ambos os métodos e as respectivas discrepâncias calculadas, para cada um dos experimentos elaborados.

Tabela 2 - Resultados dos experimentos elaborados com dados reais.

\begin{tabular}{c|c|c|c|c|c}
\hline \multicolumn{7}{c}{ POEs estimados com o método convencional } \\
\hline$\kappa_{\text {rad }}$ & $\varphi_{\text {rad }}$ & $\omega_{\text {rad }}$ & $X_{0(\mathrm{~m})}$ & $Y_{0(\mathrm{~m})}$ & $Z_{0(\mathrm{~m})}$ \\
\hline 1,9509 & 0,0015 & $-0,0124$ & 7404,23 & 3611,85 & 1652,19 \\
$\pm 0,0004$ & $\pm 0,0024$ & $\pm 0,0023$ & $\pm 1,93$ & $\pm 1,84$ & $\pm 0,55$ \\
& & & & & \\
\hline
\end{tabular}

\begin{tabular}{c|c|c|c|c|c}
\hline \multicolumn{6}{c|}{ POEs estimados com o método proposto - Experimento I } \\
\hline$\kappa_{\text {rad }}$ & $\varphi_{\text {rad }}$ & $\omega_{\text {rad }}$ & $X_{0(\mathrm{~m})}$ & $Y_{0(\mathrm{~m})}$ & $Z_{0(\mathrm{~m})}$ \\
\hline 1,9513 & 0,0010 & $-0,0054$ & 7408,54 & 3605,62 & 1652,47 \\
$\pm 0,0001$ & $\pm 0,0015$ & $\pm 0,0014$ & $\pm 1,02$ & $\pm 1,10$ & $\pm 0,18$ \\
\hline
\end{tabular}

\begin{tabular}{c|c|c|c|c|c} 
$\pm 0,0001$ & $\pm 0,0015$ & $\pm 0,0014$ & $\pm 1,02$ & $\pm 1,10$ & $\pm 0,18$ \\
\hline \multicolumn{6}{c}{ Discrepância entre o método convencional e o Experimento I } \\
\hline 0,0004 & 0,0085 & 0,007 & 4,31 & 6,23 & 0,47
\end{tabular}

POEs estimados com o método proposto - Experimento II Não encontrou solução única 
POEs estimados com o método proposto - Experimento III

\begin{tabular}{c|c|c|c|c|c}
\hline$\kappa_{\mathrm{rad}}$ & $\varphi_{\text {rad }}$ & $\omega_{\text {rad }}$ & $X_{0(\mathrm{~m})}$ & $Y_{0(\mathrm{~m})}$ & $Z_{0(\mathrm{~m})}$ \\
\hline 1,95083 & 0,0018 & $-0,0116$ & 7402,59 & 3610,81 & 1652,32 \\
$\pm 0,00006$ & $\pm 0,0002$ & $\pm 0,0002$ & $\pm 0,18$ & $\pm 0,19$ & $\pm 0,03$ \\
\hline
\end{tabular}

Discrepância entre o método convencional e o Experimento III

\begin{tabular}{l|l|l|l|l|l}
\hline 0,0008 & 0,0001 & 0,0003 & 1,64 & 1,04 & 0,13 \\
\hline
\end{tabular}

POEs estimados com o método proposto - Experimento IV

\begin{tabular}{c|c|c|c|c|c}
\hline$\kappa_{\text {rad }}$ & $\varphi_{\text {rad }}$ & $\omega_{\text {rad }}$ & $X_{0(\mathrm{~m})}$ & $Y_{0(\mathrm{~m})}$ & $Z_{0(\mathrm{~m})}$ \\
\hline 1,95023 & 0,0005 & $-0,0126$ & 7402,36 & 3611,45 & 1652,17 \\
$\pm 0,0001$ & $\pm 0,0007$ & $\pm 0,0007$ & $\pm 0,50$ & $\pm 0,50$ & $\pm 0,17$ \\
\hline
\end{tabular}

Discrepância entre o método convencional e o Experimento IV

\begin{tabular}{l|l|l|l|l|l}
\hline 0,0005 & 0,0002 & 0,0006 & 0,12 & 0,17 & 0,11 \\
\hline
\end{tabular}

\section{DISCUSSÃO DOS RESULTADOS}

Como descrito acima, quatro experimentos foram conduzidos para avaliar a potencialidade e viabilidade do método proposto para orientação indireta de imagens com dados reais. Em todos os experimentos, foram usadas edificações derivadas dos dados SLA com diferentes dimensões, orientações e distribuição geométrica. O segundo experimento não encontrou solução única para o problema, uma vez que a matriz das equações normais se apresentou mal condicionada. Os fatores que contribuíram para o problema supracitado se devem ao uso de edificações de pequeno porte e geometricamente deficientes usadas na estimativa dos parâmetros. Devido à dificuldade em selecionar, na imagem de intensidade do pulso LASER, e segmentar corretamente os tipos de edificações supracitadas, os parâmetros dos planos foram calculados com pior qualidade, ou até mesmo de forma incorreta. Desta forma, a linha reta determinada pela intersecção entre os planos segmentados de telhados adjacentes também não é confiável, além de ser de pequena dimensão, vindo a influenciar na estimativa dos parâmetros. Neste caso, deve ser investigada a influência do uso de edificações com essas características.

Dentre os experimentos que encontraram solução, o experimento I apresentou os piores resultados. O terceiro e quarto experimentos, cujas características são apresentadas na Tabela I e Figuras 4c e 4d apresentaram os melhores resultados (ver Tabela II). Os resultados encontrados com uso do conjunto de dados inerente ao experimento IV são melhores que aqueles encontrados no experimento III, porque maior quantidade de apoio de campo foi usada na estimação dos POEs. Os resultados obtidos para o experimento IV mostraram que o parâmetro de rotação omega apresentou a maior discrepância $(0,0006 \mathrm{rad})$, enquanto o maior valor de discrepância para as translações foi de $0,17 \mathrm{~m}$. Esses resultados são esperados, devido à distribuição e à orientação geométrica das edificações empregadas na estimativa.

Em todos os experimentos, a coordenada $Z_{0}$ apresentou uma boa estimativa, indicando uma das contribuições da integração de dados SLA e Fotogrametria, uma 
vez que a partir disto, se pode empregar coordenadas altimétricas (derivadas do SLA), de alta precisão, refletindo na estimativa do parâmetro supracitado.

Os desvios padrão dos parâmetros, determinados com o método proposto, revelaram valores melhores do que aqueles estimados pelo método convencional, cuja causa principal do efeito provocado é a superabundância de observações aplicada, devido à própria característica do método. Isto é, para cada edificação usada como apoio de campo existem 10 observações (no método proposto,) contra 2 observações (método convencional) de orientação indireta de imagens.

Em todos os experimentos, os POEs estimados foram influenciados por erros sistemáticos não modelados na coordenada $\mathrm{Y}$, bem como pela qualidade das coordenadas $\mathrm{X}$ e $\mathrm{Z}$ ( 0,8m e 0,25 $\mathrm{m}$ ), ambos derivados do conjunto de dados SLA. Para maiores detalhes ver Dos Santos et al. (2010).

\subsection{Vantagens e desvantagens do método}

As principais características da metodologia são: cenas de ambientes modificados pelo homem contêm uma grande quantidade de planos que podem ser facilmente segmentados; o uso de planos adjacentes tem mostrado sua potencialidade em tarefas fotogramétricas, especialmente em orientação de imagens; os planos não são considerados uma ocorrência isolada, uma vez que pela intersecção de planos adjacentes é possível extrair linhas retas, e por isso pode ser considerado geometricamente bem definido. Além disso, o número de observações é superabundante; são facilmente detectados, reconhecidos e identificados em qualquer tipo de imagem e, principalmente, com diferentes orientações. Enfim, desde que a implantação de alvos para coleta de pontos tridimensionais no espaço objeto é uma tarefa complexa e demanda esforço operacional, tempo e custo; os planos adjacentes podem ser usados como controle de campo atenuando os problemas supracitados, além de não exigir pré-sinalização de alvos.

As desvantagens do método sugerido neste trabalho são: a configuração geométrica das edificações é um fator essencial; somente telhados de duas águas podem ser empregados no método proposto, apesar de sua eficiência não depender de edificações isoladas; em áreas rurais dificilmente são encontrados edificações e a dependência de dados advindos do SLA também devem ser encarados como desvantagens no emprego do método proposto.

\section{CONCLUSÕES}

Este trabalho apresentou um método de integração de dados derivados do SLA e Fotogrametria para a orientação indireta de uma imagem digital com uso de telhados adjacentes como apoio de campo. Um modelo matemático foi desenvolvido para determinação dos parâmetros de orientação exterior de uma única imagem. Este modelo é baseado na utilização de parâmetros dos planos como apoio de campo, advindos de telhados de duas águas extraídos da imagem de intensidade do pulso laser. Foram realizados experimentos com dados reais e os resultados 
obtidos foram analisados, permitindo avaliar a viabilidade do uso do método proposto para o processo de orientação.

Para investigar a potencialidade do método proposto com dados reais, quatro experimentos foram elaborados, cujos resultados obtidos foram diretamente comparados com os resultados alcançados com o método convencional. Nos experimentos I, III e IV o método proposto se mostrou eficiente e as análises de discrepâncias revelaram que ambos os métodos são compatíveis.

O experimento II não encontrou solução e sua causa deve ser investigada. Não obstante, acredita-se que se deve evitar na solução somente o uso de edificações de pequeno porte, uma vez que neste caso, as edificações são de difícil reconhecimento, deteç̧ão e segmentação, o que implica em incoerências na determinação dos parâmetros dos planos e, consequentemente, nas linhas retas.

A contribuição mais importante do método proposto é o desenvolvimento de um modelo matemático baseado no uso de parâmetros dos planos de telhados adjacentes extraídos da imagem de intensidade do pulso laser.

Enfim, no presente caso, a análise dos resultados com dados reais permitiu concluir que o método proposto funciona adequadamente para orientação indireta de imagens, atingindo resultados compatíveis com o método convencional. A automação do método será foco de trabalhos futuros. Também serão realizados esforços para estender o modelo matemático para receber diferentes tipos de edificações e sensores de imageamento.

\section{AGRADECIMENTOS}

Os autores externam seus agradecimentos ao CNPq (Conselho Nacional de Pesquisa) pela concessão de auxílio financeiro referente ao projeto Jovem Pesquisador no. Processo 570316/2008-1, ao LACTEC e à Empresa AGRITEC/A.S., por disponibilizarem os dados derivados do sistema LiDAR e as imagens digitais adquiridas com a câmara digital DSC-F717.

\section{REFERÊNCIAS BIBLIOGRÁFICAS}

DALMOLIN, Q., SANTOS, D. R., DELARA, R., BAHR, H. P., O uso de feições retas extraídas do MDT gerado pelo sistema de varredura LASER como apoio para o cálculo da resseção espacial de imagens digitais. Boletim de Ciências Geodésicas, Curitiba, v. 11, n. 1, p.117-140, 2005.

DOS SANTOS, D. R., DAL POZ, A. P., DALMOLIN, Q., Indirect orientation of images using control points extracted by the means of monoplotting model. The Photogrammetric Journal of Finland, 22(1), pp. 25-43, 2010.

HABIB, A. F., Motion parameters estimation by tracking stationary threedimensional straight lines in image sequences. ISPRS Journal of Photogrammetric and Remote Sensing, 53 (2), pp. 174-182, 1999.

HABIB A. F., MORGAN M., LEE Y. R., Bundle Adjustment with self-calibration using straight lines. Photogrammetric Record, 17 (100), pp. 635-650, 2002. 
HABIB A. F., GHANMA, M. S., HIN, C. J., MitishitA, E. A., Alternative approaches for utilizing ALS data as a source of control information for photogrammetric models. International Archives of XXth ISPRS Congress, Istanbul, Turkey, Commission I: 6p. (CD-ROM), 2004.

HABIB A. F., GHANMA, M. S., MITISHITA, E. A., Photogrammetric georeferencing using ALS linear and areal features. Korean Journal of Geomatics, 5(1): pp. 1-13, 2005a.

HABIB A. F., GHANMA, M. S., MORGAN, M. F., AL-RUZOUQ, R., Photogrammetric registration using linear features. Photogrammetric Engineering and Remote Sensing, 71(6): pp. 699-707, 2005b.

HABIB A. F., BANG, K. I., ALDELGAWY, M. SHIN, S. W., KIN, K. O., Integration of photogrammetric and ALS in a multi-primitive triangulation procedure. ASPRS Annual Conference, 7-11, 2007.

LUGNANI, J. B., Using Digital Entities as Control. 1980. 159f. PhD thesis. Department of Surveying Engineering. University of New Brunswick.

MULAWA, D. C., MIKHAIL, E. M. Photogrammetric Treatment of linear features. In: Proceedings of 16th International Congress of Photogrammetry and remote Sensing. Kyoto, Commission III, pp. 383-393,1988.

TOMMASELLI, A. M. G., LUGANANI, J. B., An Alternative Mathematical Model to the Colinearity Equation Using Straight Features. Proceedings of the International Society for Photogrammetry and Remote Sensing, Kyoto, Commission III, pp. 756-774, 1988.

TOMMASELLI, A. M. G., TOZZI, C. A., Recursive approach to space resection using straight lines. Photogammetric Engineering and Remote Sensing, 62 (1), pp. 55-66, 1996.

(Recebido em julho de 2011. Aceito em fevereiro de 2012.) 